\title{
MECHANICAL HOMOGENEOUS CONTINUOUS DYNAMICAL SYSTEMS HOLOR ALGEBRA - STEADY-STATE ALTERNATING VELOCITY ANALYSIS
}

\author{
B. FIJAŁKOWSKI \\ Institute of Technology \\ State Higher Vocational School in Nowy Sącz \\ 33-300 Nowy Sącz, ul. Zamenhofa 1a, POLAND \\ E-mail:pmfijalk@cyf-kr.edu.pl
}

\begin{abstract}
In this article, a new mathematical representation of the sinusoidal alternating velocity, force and power by means of some complex quantities, termed 'holors' is proposed. The word holor is a term to describe a mathematical entity that is made up of one or more independent quantities, and includes complex numbers, scalars, vectors, matrices, tensors and other hypernumbers. Holors, thus defined, have been known for centuries but each has been developed more or less independently, accompanied by separate nomenclature and theory.
\end{abstract}

Key words: force, velocity, power, holor.

\section{Introduction}

The steady-state behaviour's laws of alternating velocity (AV) mechanical homogeneous continuous dynamical systems that are energised by sinusoidal AV energy sources are an important area of study for several reasons. First, the generation, transmission, distribution, and consumption of mechanical energy occur under essentially sinusoidal AV steady-state conditions. Second, it is possible to predict the behaviour of AV mechanical homogeneous continuous dynamical systems to non-sinusoidal energy sources if the sinusoidal behaviour is understood. Third, it is often easier to carry out the design of AV mechanical homogeneous continuous dynamical systems based on a steady-state sinusoidal behaviour. Thus, design specifications are spelled out in terms of a desired steady-state sinusoidal response. The AV mechanical homogeneous continuous dynamical system is designed to meet these sinusoidal characteristics. If these specifications are satisfied, the designer knows that the AV mechanical homogeneous continuous dynamical system will respond satisfactorily to non-sinusoidal inputs.

\section{Sinusoidal alternating-velocity holor algebra - steady-state analysis}

\subsection{The sinusoidal AV energy source}

A sinusoidal AV 'mechanical energy source' produces mechanical energy. It may act as a sinusoidal 'force source' (independent or depended) that produces a force that varies sinusoidally with time, or -- as a sinusoidal 'velocity source' (independent or dependent) that produces a velocity that also varies sinusoidally with time.

One can express a sinusoidally varying function using either the sine function or the cosine function. There is no clear-cut choice for either function. Although either works, equally well in sinusoidal steadystate analysis, it is important to recognise that both functional waveforms cannot be used simultaneously. The sine function will be used throughout the discussion below. 


\subsection{The generation of alternating velocity}

A velocity or force is termed 'alternating' if it periodically changes its sense of direction and magnitude. The complete period of a velocity variation is usually termed simply the 'period' $T$ of the alternating velocity, and the number of such periods per second

$$
f=T^{-1},
$$

is termed the 'frequency'. The unit of frequency is $\left[s^{-1}\right]$ cycles per second [cps], and is sometimes, especially in Europe, termed hertz [Hz].

A voice-coil AV electro-mechanical (EM) actuator shown in Fig.1 [1] may be used for a generation of alternating velocity.

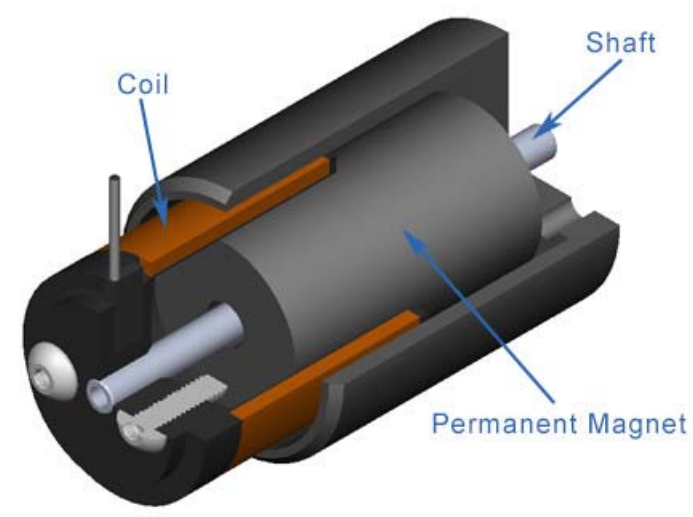

Fig.1. Schematic cross-section of a voice-coil AV EM actuator [1].

An AV EM actuator is a transducer that converts an electrical signal to a desired physical quantity. An active force control AV EM actuator modifies a force by providing an electronically controllable disturbance. The field of active force control has witnessed explosive growth in the variety of AV EM actuators, which is an evidence of both the importance and challenges associated with an AV EM actuator design. A linear EM motor that converts electrical energy into mechanical energy (force) powers an AV EM actuator.

A voice-coil AV EM actuator is ideal for short stroke (less than $0.5 \mathrm{~m}$ ) closed loop servo applications. It has very low electrical and mechanical time constants. The low moving mass allows for high accelerations of light payloads. This AV EM actuator is wound in such a way that no com-mutation is required for motion to occur. The result is a much simpler and more reliable system [1].

Coupling the AV EM actuator with a bearing system, position feedback device, linear servo amplifier and motion controller yields a system that is capable of intricate position, velocity, and acceleration control. This AV EM actuator can also be used for precise force control because of the linear force versus velocity characteristics.

A voice coil AV EM actuator operates on the principal of the Lorentz force equation (LFE).

$$
F=B \times I
$$

where: $F-$ force $[N]$;

$B$ - magnetic flux density $[T]$ '

$I$ - electrical current $[A]$. 
Simply stated, a current carrying conductor placed in a magnetic field will have a force exerted upon it. This force is proportional to the sense of direction and magnitude of the electrical current and the magnetic flux density. Since the interior permanent magnet (IPM) magnetic flux density is fixed, the sense of direction of the linear displacement depends on the polarity of the input electrical current. The amount of force that is produced is directly proportional to the magnitude of the input electrical current. Higher forces can be achieved by increasing the diameter. This type of AV EM actuator can be designed with large radial clearances to ensure no contact between the coil and magnet assembly if a tilt or radial movement is required. It should be noted that for an AV EM actuator, a longer stroke is available but the efficiency decreases as the stroke increases. If higher efficiency is desired for a longer-stroke AV EM actuator, the coil assembly can be wound as a multi-phase coil and then it will have to be commutated. The voice-coil AV EM actuator in open-loop mode can be operated and is ideal for controlled sinusoidal motion applications ( 1 to $1000 \mathrm{~Hz}$ for open-loop applications and 1 to $125 \mathrm{~Hz}$ for closed-loop applications). The voice-coil AV EM actuator is specifically designed for high precision, push-rod applications where positioning accuracy, high alternating velocity and high alternating force capacities are necessary. One move of a voice-coil AV EM actuator's mover (forcer) corresponds to one cycle of an AV energy-source force in the voice-coil phase windings, and if the mover makes $n$ motions per second $[\mathrm{mps}]$, the frequency of the induced energy-source force is

$$
f=n
$$

Since the angular velocity of the AV EM actuator is, in this case,

$$
\omega=2 \pi n
$$

there exists a simple relationship between it and the angular frequency of the induced energy-source force

$$
\omega=2 \pi f=2 \pi / T \text {. }
$$

For an AV E-M actuator with $p$ pairs of magnetic poles

$$
f=p n
$$

Independently of the AV EM actuator construction, the value $\omega$ determined from Eq.(2.3) is termed the 'angular frequency' of alternating velocity. Thus, the angular frequency is the angular velocity of a two-pole AV EM actuator, the energy-source force of its has a frequency $f$. The unit of angular frequency is $\left[s^{-1}\right]$.

For instance, the standard 125 or $1000 \mathrm{~Hz}$ frequency corresponds to an angular frequency

$$
\omega=2 \pi f=2 \pi 125=785 \mathrm{~s}^{-1},
$$

or

$$
\omega=2 \pi f=2 \pi 1000=6283 \mathrm{~s}^{-1} .
$$

The alternating velocity of a vibrating object varies from zero to a maximum during each cycle of vibration. It moves fastest as it passes through its natural stationary position to an extreme position. The vibrating object slows down as it approaches the extreme, where it stops and then moves in the opposite sense of direction through the stationary position toward the other extreme. The speed of vibration is expressed in units of metres per second $[\mathrm{m} / \mathrm{s}]$. 
The speed is a scalar quantity that refers to 'how fast an AV EM actuator's shaft or mover is moving.' Velocity is a holor or vector quantity that refers to 'the rate at which an AV EM actuator's shaft or mover changes its displacement or position.'

\subsection{Sinusoidal alternating velocity}

The alternating energy-source force of an AV EM actuator and the alternating velocity can have highly diversified variation waveforms, which largely depend on constructional factors, for instance, the shape of the mover (forcer) magnetic-pole pieces.

The majority of all periodic waveforms are the sine or cosine waveforms, which are the only periodical functions having derivatives like themselves. The technical significance of this fact is that the shape of the waveforms of forces and velocities (Fig.2) is the same in all components of an AV mechanical homogeneous continuous dynamical system, i.e., sinusoidal or harmonic.

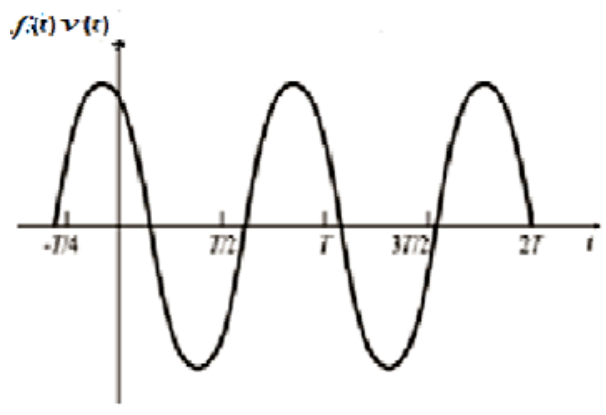

Fig.2. Sinusoidal waveform of the force or velocity $[2,3]$.

Analytical expressions for the sinusoidal functions of instantaneous values of the forces and velocities are $[1,2]$

$$
\begin{aligned}
& f(t)=F_{\text {max }} \sin \left(\omega t+\psi_{f}\right)=\sqrt{2} F_{r m s} \sin \left(\omega t+\psi_{f}\right), \\
& v(t)=V_{\text {max }} \sin \left(\omega t+\psi_{v}\right)=\sqrt{2} V_{r m s} \sin \left(\omega t+\psi_{v}\right)
\end{aligned}
$$

where the small letters $f(t)$ and $v(t)$ denote the instantaneous values of force and velocity. i.e., their values at an arbitrary time $t$; the capital letters $F_{\max }$ and $V_{\max }$ denote the corresponding amplitudes, i.e., the maximum values, $F_{r m s}=F$ and $V_{r m s}=V$ denote the corresponding effective values; and $\psi_{f}$ and $\psi_{v}$ denote the corresponding phase angles depending on the values of force and velocity at the instant chosen as the time origin $(t=0)$.

If one takes the initial time to be that when the velocity passes through its maximum, $\sin \left(\omega t+\psi_{i}\right)$ at $t=0$ must be equal to unity

$$
\sin \left(\omega t+\psi_{v}\right)=1 \quad \text { moreover, } \quad \psi_{v}=\pi / 2 .
$$

The various stages of processes described by sinusoidal functions, namely: growth, decay, passage through zero or a maximum, and so on, are termed phases. The phase of a process at any time $t$ is defined by the angle $\omega t+\psi$ of the sine and is termed the phase angle. 
The angle $\psi$ which determines the phase at a given initial instant $(t=0)$, is termed the initial phase, and the difference between the initial phases $\psi_{f}$ and $\psi_{v}$ of the two functions is termed the phase difference or phase shift.

\section{Sinusoidal force and velocity waveforms - holor representation}

In an AV mechanical homogeneous continuous dynamical system one shall usually deal with sinusoidal force and velocity waveforms that are all of the same frequency but which may be shifted from each other in time. We shall start by discussing sinusoidal force and velocity waveforms. A very simple sinusoidal force and velocity instantaneous values with using of the trigonometric identity are $[2,3]$

$$
\begin{aligned}
& f(t)=\sqrt{2} F \sin \left(\omega t+\psi_{f}\right)=\sqrt{2} F\left[\left(\sin \omega t \cos \psi_{f}+\cos \omega t \sin \psi_{f}\right)\right], \\
& v(t)=\sqrt{2} V \sin \left(\omega t+\psi_{v}\right)=\sqrt{2} V\left[\left(\sin \omega t \cos \psi_{v}+\cos \omega t \sin \psi_{v}\right)\right],
\end{aligned}
$$

where: $\psi_{f}$ - the force phase angle;

$\psi_{v}$ - the velocity phase angle.

Now, one must be able to convert Eq.(3.1) of the forms

$$
\begin{aligned}
& f(t)=\sqrt{2}\left[F_{s} \sin \omega t+F_{c} \cos \omega t\right], \\
& v(t)=\sqrt{2}\left[V^{s} \sin \omega t+V^{c} \cos \omega t\right] .
\end{aligned}
$$

Then, comparing Eqs (3.2) with Eqs (3.1), one obtains

$$
F_{c}=F \cos \psi_{f}, \quad F_{s}=F \sin \psi_{f} .
$$

The quantities $F_{c}$ and $F_{s}$ are independent elements (merates) of an effective value of the force holor

$$
\boldsymbol{F}_{i}=\left(F_{c}, F_{s}\right)
$$

The above written holor fully represents a sinusoidal force. Analogically, a sinusoidal velocity can be represented by an effective value of the velocity holor

$$
\boldsymbol{V}^{j}=\left(V^{c}, V^{s}\right)
$$

where

$$
\boldsymbol{V}^{c}=V \cos \psi_{v}, \quad V^{s}=V \sin \psi_{v} .
$$

Thus, the sinusoidal force holor $\boldsymbol{F}_{i}$ and/or velocity holor $V^{j}$ are defined as mathematical entities or wholes built up of two independent elements (merates) $F_{c}$ and $F_{s}$ as well as $V^{c}$ and $V^{s}$ that carry the 
effective values and phase angles information of sinusoidal functions to the doublet (dipole) functions of two independent quantities that cannot be directly added.

Due to the fact that independent elements of the force holor as well as the velocity holor depend only on effective values and phase angles of these quantities, then force holor and velocity holor cane represented in the same way as in complex numbers, namely:

$$
\boldsymbol{F}_{i}=F \angle \psi_{f},
$$

and

$$
\boldsymbol{V}^{j}=V \angle \psi_{v} .
$$

As in Eqs (3.7) to (3.8) a holor quantity will be represented throughout the text by a boldface letter. In studying Eqs (3.7) and (3.8) observe that the shorthand abbreviation $\angle$ in the angle notation of the phase angles belongs to holors that carry the effective values and phase angles of the given sinusoidal functions. This notation will used extensively in what follows. These holors are by definition the holor representation', or 'holor transform', of the given sinusoidal functions.

Thus, the holor transform transfers the sinusoidal function from the time domain to the holor domain. Thus far, moving from the sinusoidal function to its holor transform has been emphasized. However, it should be apparent that one could also reverse the process. That is, given the holors one can write the expressions for the sinusoidal functions.

Thus, if one is given that $\boldsymbol{F}_{i}=F \angle \psi_{f}$ and $\boldsymbol{V}^{j}=V \angle \psi_{v}$, the expressions for

$$
f(t)=\sqrt{2} F \sin \left(\omega t+\psi_{f}\right) \quad \text { and } \quad v(t)=\sqrt{2} V \sin \left(\omega t+\psi_{v}\right)
$$

since it has been decided to use the sine function for all sinusoids.

Observe that one cannot deduce the value of $\omega$ from the holor. The holors carry only magnitude of the effective values and phase angles information. The step of going from the holor transform to the timedomain expression is referred to as finding the 'inverse' holor transform and is formalised by the equation

$$
\begin{aligned}
& \mathbb{H}^{-1}\left\{F \angle \psi_{f}\right\}=\sqrt{2}\left\{F \angle \omega t+\psi_{f}\right\} \\
& \mathbb{H}^{-1}\left\{V \angle \psi_{i}\right\}=\sqrt{2}\left\{V \angle \omega t+\psi_{v}\right\}
\end{aligned}
$$

where the notations $\mathbb{H}^{-1}\left\{F \angle \psi_{v}\right\}$ and $\mathbb{H}^{-1}\left\{V \angle \psi_{i}\right\}$ are read as the inverse transform' of $F \angle \psi_{f}$ and $V \angle \psi_{v}$.

Equation (3.9) tells us that to find the inverse holor transform one multiplies the holors by $\sqrt{2}\{F \angle \omega t\}$ and $\sqrt{2}\{V \angle \omega t\}$ and then to extract the second merates $F_{s}$ and $V^{s}$ of the products, respectively. The holor transform is so useful in mechanical system analysis because it reduces the problem of finding the maximum amplitude and phase angle of the steady-state sinusoidal response to the algebra of holors. In a linear mechanical continuous dynamical system driven by sinusoidal energy sources, the steady-state response is also sinusoidal. The holor transform is also useful in mechanical system analysis because it applies directly to the sum of sinusoidal functions. Since mechanical continuous dynamical system analysis involves summing forces and velocities. The importance of this observation is obvious. The holor representation of the sum is the sum of the holors of the individual independent elements (merates). 
The superiority of the holor approach to adding sinusoidal functions should be apparent. One should note that the holor approach requires the ability to move back and forth between the polar and rectangular forms.

\section{Passive AV mechanical homogeneous continuous dynamical system components in the holor domain}

The systematic application of the holor transform in the AV mechanical homogeneous continuous dynamical system analysis requires two steps. First, one must establish the relationship between the velocity holor and the force holor of the passive AV mechanical homogeneous continuous dynamical system's components. Second, one must develop the holor-domain version of Kirchhoff's Laws, which will be discussed in the next section. In this section, the relationship between the velocity holor and the force holor at the conductive collectors of the damper, spring, and mass will be established. We begin with the damper. The passive sign convention will be used in all the derivations. It follows directly from Ohm's Law that if the velocity in a damper varies sinusoidally with the time, that is, if $v(t)=\sqrt{2} V \sin \left(\omega t+\psi_{v}\right)$, then the force at the conductive collectors of the damper will be

$$
f(t)=B \sqrt{2} V \sin \left(\omega t+\psi_{v}\right)
$$

where $V_{\max }=\sqrt{2} V$ is the maximum amplitude of the velocity in metres per second $\left[\mathrm{m} \mathrm{s}^{-1}\right]$; and $\psi_{v}$ is the phase angle of the velocity.

The holor transform of this force holor is

$$
\boldsymbol{F}_{i}=\boldsymbol{B}_{i j} V \angle \psi_{v}
$$

However, $V \angle \psi_{v}$ is the holor representation of the sinusoidal velocity holor; thus, one can write Eq.(4.2) as

$$
\boldsymbol{F}_{i}=\boldsymbol{B}_{i j} \boldsymbol{V}^{j}=\left\|\begin{array}{cc}
B & 0 \\
0 & B
\end{array}\right\|\left\|\begin{array}{l}
V^{c} \\
V^{s}
\end{array}\right\|
$$

which implies that the force holor at the conductive collectors of a damper is simply the damper holor $\boldsymbol{B}_{i j}$ times the velocity holor.

The AV mechanical homogeneous continuous dynamical system diagram for a damper in the holor domain is shown in Fig.3.

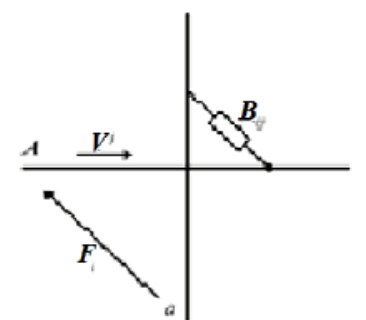

(a)

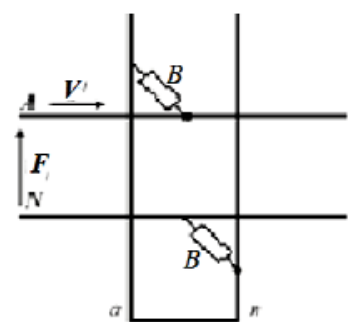

(b)

Fig.3. The holor domain equivalent AV mechanical homogeneous continuous dynamical system for a damper $[2,3]$. 
However, $F \angle \psi_{f}$ is the holor representation of the sinusoidal force holor; thus, one can write Eq.(4.2) as

$$
\boldsymbol{V}^{j}=\boldsymbol{S}^{j i} \boldsymbol{F}_{i}=\frac{\delta_{k}^{i}}{\boldsymbol{B}_{j k}} \boldsymbol{F}_{i}=\left\|\begin{array}{cc}
S & 0 \\
0 & S
\end{array}\right\| F_{c} \|
$$

which implies that the velocity holor at the conductive collectors of a stimulus is simply the stimulus holor $\boldsymbol{S}^{j i}$ times the force holor. This phase relationship is shown in Fig.4.

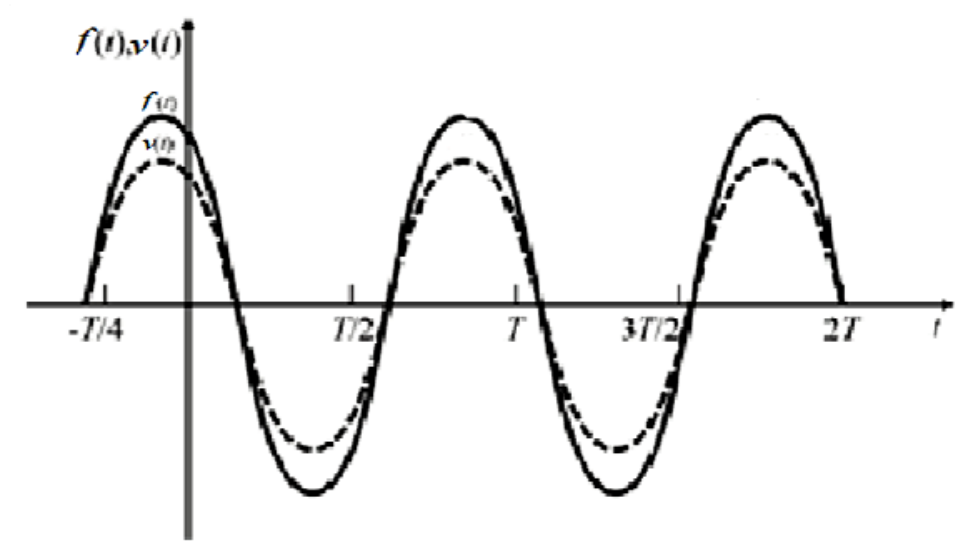

Fig.4. A damper AV mechanical-homogeneous-continuous-dynamical-system's dynamical component carrying a sinusoidal alternating velocity $[2,3]$.

The signals are said to be in time phase because they both reach corresponding values on their respective waveforms at the same time (for example, they are at their positive maxima at the same instant).

The relationship between the velocity and force at the conductive connectors of the spring is derived by assuming a sinusoidal velocity and using $K d i / d t$ to establish the corresponding force. Thus, given $v(t)=\sqrt{2} V \sin \left(\omega t+\psi_{v}\right)$, one sees that the expression for the force is

$$
f(t)=K \frac{d v(t)}{d t}=\omega K \sqrt{2} V \cos \left(\omega t+\psi_{v}\right)
$$

Now one rewrites Eq.(4.5) using the sine function

$$
f(t)=K \frac{d v(t)}{d t}=\omega K \sqrt{2} V \sin \left(\omega t+\psi_{v}+\pi / 2\right) .
$$

The holor representation of the force holor given by Eq.(4.5) is

$$
\boldsymbol{F}_{i}=\boldsymbol{X}_{i j}^{K} \boldsymbol{V}^{j}=\left\|\begin{array}{cc}
0 & -\omega K \\
\omega K & 0
\end{array}\right\| V \angle \psi_{i}+\pi / 2=\left\|\begin{array}{cc}
0 & -\omega K \\
\omega K & 0
\end{array}\right\| \boldsymbol{V}^{j} .
$$


From Eq.(4.7), one sees that the force holor at the conductive conductors of a spring equals the spring-reactance holor $\boldsymbol{X}_{i j}^{K}$ times the velocity holor.

The holor domain equivalent AV mechanical homogeneous continuous dynamical system is shown in Fig.5. The phase relationship between the velocity and force at the conductive conductors of a spring is contained in Eq.(4.7) from which one see that the force and velocity will be out of phase by exactly $\pi / 2$.

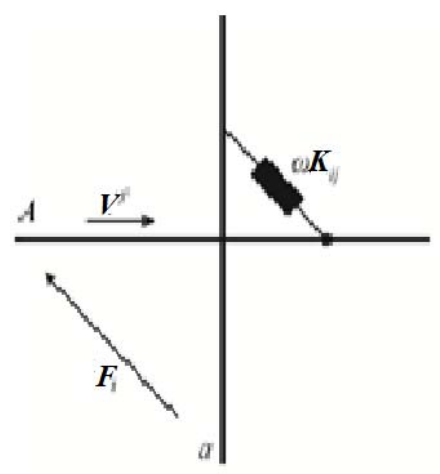

(a)

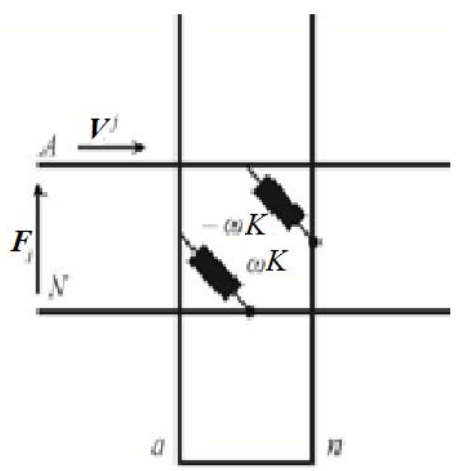

(b)

Fig.5. The holor domain equivalent AV mechanical homogeneous continuous dynamical system for a spring $[2,3]$.

In particular, the force will lead the velocity by $\pi / 2$ or what is equivalent, the velocity will lag the force by $\pi / 2$. This concept of 'force leading velocity' or 'velocity lagging force' is made clear by the plot shown in Fig.6, where one notes that as time increases from zero, the force comes to a particular instantaneous value on the sine function ahead of the velocity. For instance, it reaches its negative peak exactly $\pi / 2$ before the velocity reaches its negative peak.

The same observation can be made with respect to the 'zero-going positive' crossing or the positive peak. Thus, one says that the force 'leads' the velocity by $\pi / 2$ or, what is equivalent, the velocity 'lags' behind the force by $\pi / 2$.

The phase shift can also be expressed as a period $T$ in seconds $[s]$. A phase shift of $\pi / 2$ corresponds to one fourth of a period, hence the force 'leads' the velocity by $T / 4$, or $1 / 4 f s$.

The relationship between the velocity and force at the conductive conductors of a mass follows directly from the derivation of Eq.(4.8).

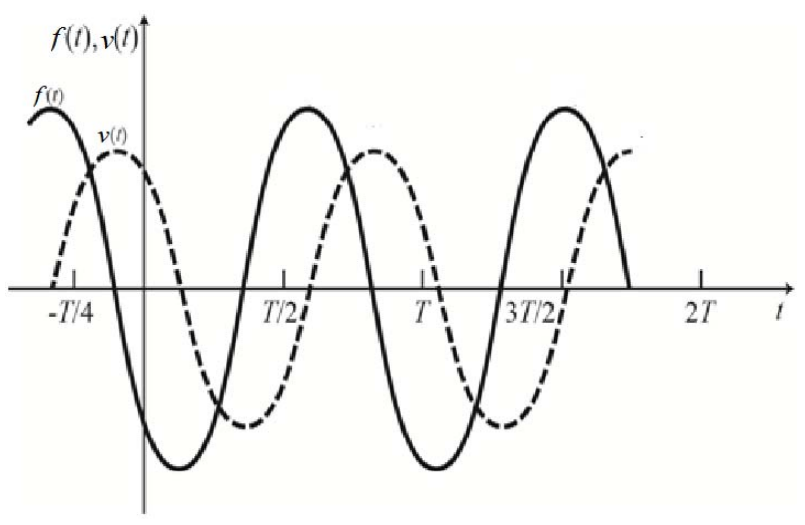

Fig.6. A spring AV mechanical-homogeneous-continuous-dynamical-system's dynamical component carrying a sinusoidal alternating velocity $[1,2]$. 
That is, if one notes that for a mass

$$
f(t)=m \frac{d v(t)}{d t}
$$

and then assume

$$
f(t)=\sqrt{2} F \sin \left(\omega t+\psi_{f}\right),
$$

it follows that

$$
\boldsymbol{V}^{j}=\boldsymbol{Y}_{m}^{j i} \boldsymbol{F}_{i}=\frac{\delta_{k}^{i}}{\boldsymbol{Z}_{j k}^{m}} \boldsymbol{F}_{i}=\left\|\begin{array}{c}
V^{c} \\
V^{s}
\end{array}\right\|=\left\|\begin{array}{cc}
0 & -\omega m \\
\omega m & 0
\end{array}\right\|\left\|\begin{array}{c}
F_{c} \\
F_{s}
\end{array}\right\|
$$

Now, if we write Eq.(4.8) for the force holor as a function of the velocity, then we obtain

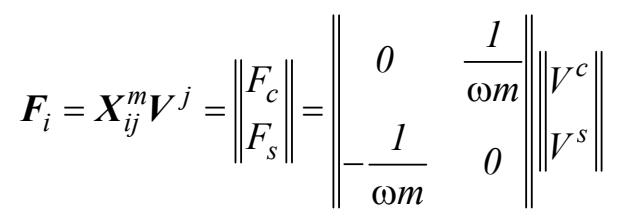

From Eq.(4.10), we see that the equivalent AV mechanical continuous dynamical system for the mass in the holor domain is as shown in Fig.7.

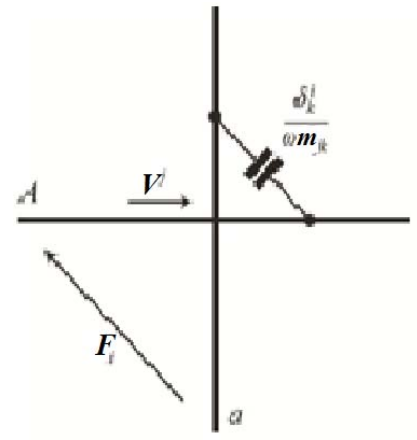

(a)

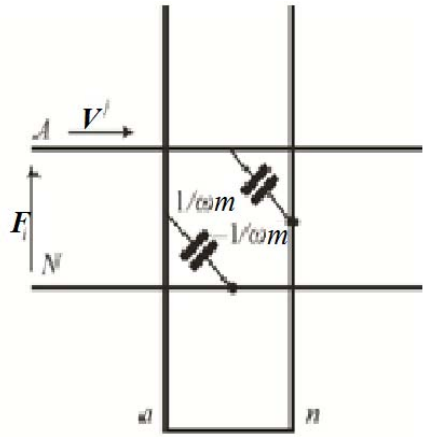

(b)

Fig.7. The holor domain equivalent AV mechanical continuous dynamical system for a mass $[2,3]$.

The force holor across the conductive conductors of a mass will 'lag' behind the mass velocity holor by exactly $\pi / 2$.

One can easily see this by rewriting Eq.(4.10) as

$$
\boldsymbol{F}_{i}=\boldsymbol{X}_{i j}^{m} \boldsymbol{V}^{j}=\left\|\begin{array}{l}
F_{c} \\
F_{s}
\end{array}\right\|=\left\|\begin{array}{cc}
0 & \frac{1}{\omega m} \\
1 & 0
\end{array}\right\|\left\|\begin{array}{l}
V^{c} \\
V^{s}
\end{array}\right\|
$$

where

$$
\boldsymbol{V}^{j}=V^{c} \quad V^{s^{T}}=V \angle \psi_{v}-\pi / 2
$$


The alternative way to express the phase relationship contained in Eq.(4.11) is to say that the velocity holor 'leads' the force holor by $\pi / 2$.

The phase relationship between the velocity holor and force holor at the conductive conductors of a mass is shown in Fig.8.

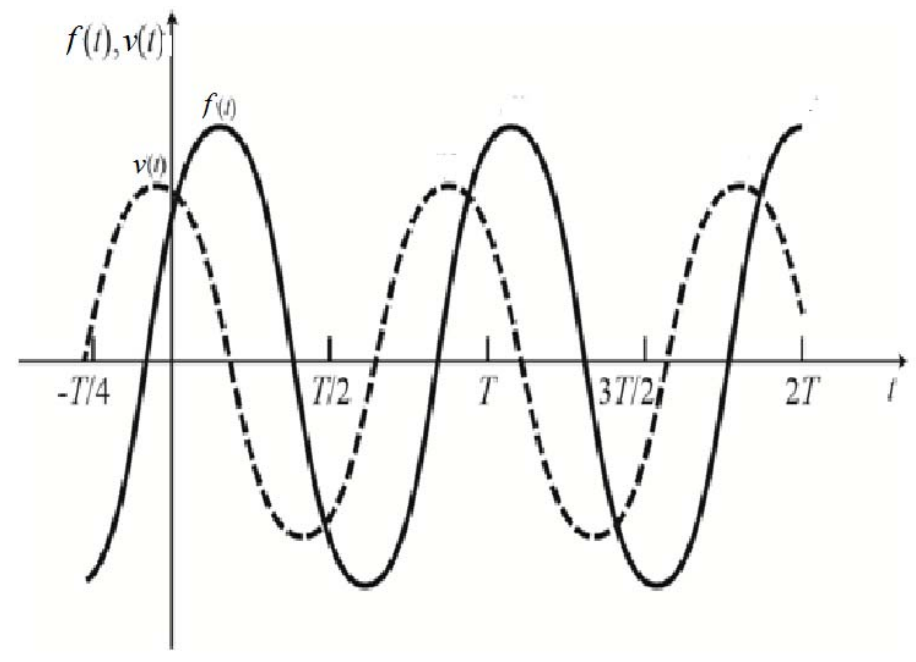

Fig.8. A mass AV mechanical-homogeneous-continuous-dynamical-system's dynamical component carrying a sinusoidal alternating velocity $[2,3]$.

Our discussion of the passive AV mechanical-homogeneous-continuous-dynamical-system's dynamical components is concluded in the holor domain with a very important observation.

When one compares Eqs (4.3), (4.6), and (4.11), one notes that they are all of the form

$$
\boldsymbol{F}_{i}=\boldsymbol{Z}_{i j} \boldsymbol{V}^{j}
$$

where $\boldsymbol{Z}_{i j}$ represents the 'impedance holor matrix' of the AV mechanical-continuous-dynamical system components.

Thus, the impedance of a damper is $B$, the impedance of a spring is $\omega K$, and the impedance of a mass is $1 / \omega m$. In all cases, the impedance holor is measured in kilograms per second $\left[\mathrm{kg} \mathrm{s}^{-1}\right]$.

The concept of impedance is very powerful in sinusoidal steady-state analysis and much more will be said about its usefulness in subsequent sections.

The term 'spring reactance' is used to describe the product $\omega K$. The reactance of a spring is $\omega K$ $\left[\mathrm{kg} \mathrm{s}^{-1}\right]$, whereas the impedance of a spring is $\omega K\left[\mathrm{~kg} \mathrm{~s}^{-1}\right]$.

The term 'mass reactance' is used to describe the quantity $-1 / \omega m$. The reactance of a mass is $1 / \omega m$ $\left[\mathrm{kg} \mathrm{s}^{-1}\right]$, whereas the impedance of a mass is $-1 / \omega \mathrm{m}\left[\mathrm{kg} \mathrm{s} \mathrm{s}^{-1}\right]$.

Let us make one final comment about the impedance holor. Note that although the impedance holor is a bivalent holor it is a matrix $\boldsymbol{Z}_{i j}$.

Finally, mind that if the reference sense of direction for the velocity holor in a passive AV mechanical-homogeneous-continuous-dynamical-system's dynamical component is in the sense of direction of the force holor rise across the dynamical component, a minus sign must be inserted into the equation that relates the force holor to the velocity holor. 


\section{An impedance - holor representation}

Applying Kirchhoff's Second Law (KSL) to the $B-K-m$ AV mechanical homogeneous continuous dynamical system shown in Fig.9 yields

$$
f(t)=B v(t)+K \frac{d v(t)}{d t}+\frac{1}{m} \int_{0}^{t} v(t) d t
$$

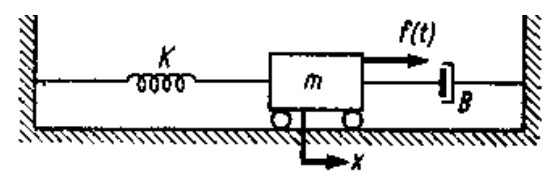

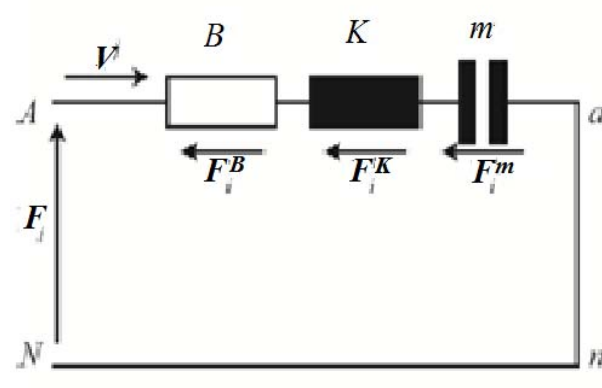

(a)

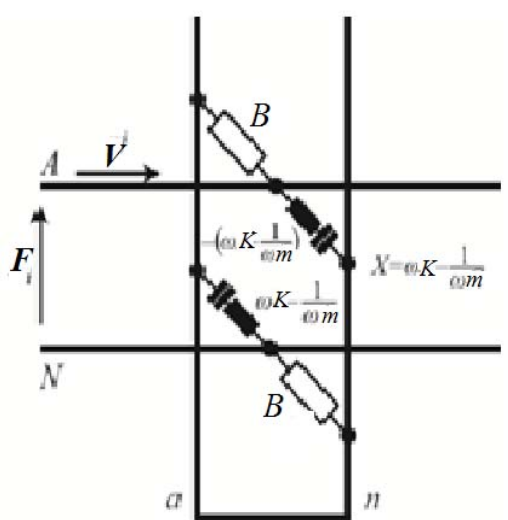

(b)

Fig.9. Holor equivalent MCM of the $B-K-m$ AV mechanical homogeneous continuous dynamical system $[2,3,4]$.

Substituting an instantaneous value of the velocity from Eqs (3.1) to Eq.(5.1) one has

$$
v(t)=\sqrt{2} V\left[\left(B \cos \psi_{v}-X \sin \psi_{v}\right) \sin \omega t+\left(B \sin \psi_{v}+X \cos \psi_{v}\right) \cos \omega t\right]
$$

where, the passive reactance is given as

$$
X=\omega K-1 / \omega m \text {. }
$$

Substituting an instantaneous value of the force from Eq.(4.1) to Eqs (3.6) one has

$$
\begin{aligned}
& \left(F \cos \psi_{f}-B V \cos \psi_{v}+X V \sin \psi_{v}\right) \sin \omega t+ \\
& +\left(F \sin \psi_{f}-B V \sin \psi_{v}-X V \cos \psi_{v}\right) \cos \omega t=0 .
\end{aligned}
$$


But $\sin \omega t$ and $\cos \omega t$ are the independent linear functions, one has

$$
\begin{aligned}
& F \cos \psi_{f}=B V \cos \psi_{v}-X V \sin \psi_{v}, \\
& F \sin \psi_{f}=B V \sin \psi_{v}+X V \cos \psi_{v} .
\end{aligned}
$$

Comparing Eqs (3.4) and (3.7) with Eqs (5.5) and (5.6) one obtains the relationship between independent elements (merates) of the force holor $\boldsymbol{F}_{i}$ and independent elements (merates) of the velocity holor $\boldsymbol{V}^{j}$, namely

$$
F_{c}=B V^{c}-X V^{s}, \quad F_{s}=X V^{c}+B V^{s}
$$

Assuming that

$$
Z_{c c}=B, \quad Z_{c s}=-X, \quad Z_{s c}=X, \quad Z_{s s}=B
$$

Equation (5.7) can be rewritten as

$$
F_{c}=Z_{c c} V^{c}-Z_{c s} V^{s}, \quad F_{s}=Z_{s c} V^{c}+Z_{s s} V^{s} .
$$

Thus, the relationship between the velocity holor $\boldsymbol{V}^{j}$ and the force holor can be described by an expression

$$
\boldsymbol{F}_{i}=\boldsymbol{Z}_{i j} \boldsymbol{V}^{j}
$$

which defines a new bivalent holor $\boldsymbol{Z}_{i j}$, termed the bivalent impedance holor.

The impedance holor written in a general form establishes a matrix

$$
Z_{i j}=\left\|\begin{array}{cc}
Z_{c c} & -Z_{c s} \\
Z_{s c} & Z_{s s}
\end{array}\right\|
$$

In particular circumstances:

a) For a series $B-K-m$ AV mechanical homogenous continuous dynamical system, the impedance holor $\boldsymbol{Z}_{i j}$ well known in matrix form of the impedance holor matrix $[Z]$, namely $[2,3]$ :

$$
\boldsymbol{Z}_{i j}=\left\|\begin{array}{cc}
B & -X \\
X & B
\end{array}\right\|=\left\|\begin{array}{cc}
B & 1 / \omega m-\omega K \\
\omega K-1 / \omega m & B
\end{array}\right\|
$$

where

$$
X=\omega K-1 / \omega m
$$

b) For an ideal active damper $B$, the passive reactance $X=0$, hence

$$
\boldsymbol{Z}_{i j}=\left\|\begin{array}{cc}
B & 0 \\
0 & B
\end{array}\right\|=B\left\|\begin{array}{cc}
1 & 0 \\
0 & 1
\end{array}\right\|=B 1
$$


c) For a series $B-K$ AV mechanical homogeneous continuous dynamical system, the passive reactance $X=\omega K$, hence

$$
Z_{i j}=\left\|\begin{array}{cc}
B & -\omega K \\
\omega K & B
\end{array}\right\|
$$

d) For a series $B-m$ AV mechanical homogeneous continuous dynamical system, the passive reactance $X=1 / \omega m$, hence

$$
Z_{i j}=\left\|\begin{array}{cc}
B & 1 / \omega m \\
-1 / \omega m & B
\end{array}\right\| .
$$

Applying the holor algebra laws, which govern the bivalent holors one obtains as well, the impedance holors for other much-complicated AV mechanical homogeneous continuous dynamical systems.

\section{An admittance - holor representation}

Equation (5.9) for the velocity holor can be also rewritten in a form

$$
\boldsymbol{V}^{j}=\boldsymbol{Y}^{j i} \boldsymbol{F}_{i}
$$

where, the admittance holor $\boldsymbol{Y}^{j i}$ is the inverse of impedance holor

$$
\boldsymbol{Y}^{i j} \boldsymbol{Z}_{j k}=\delta_{k}^{i}
$$

where, $\delta_{k}^{j}$ is a Kronecker delta.

For instance, for a series $B-K-m \mathrm{AV}$ mechanical homogeneous continuous dynamical system

$$
\boldsymbol{Y}^{j i}=\left\|\begin{array}{cc}
Y^{s s} & Y^{s c} \\
-Y^{c s} & Y^{c c}
\end{array}\right\|=\left\|\begin{array}{cc}
G & H \\
-H & G
\end{array}\right\|=\frac{1}{\left(B^{\wedge} 2\right)+\left(X^{\wedge} 2\right)}\left\|\begin{array}{cc}
B & X \\
-X & B
\end{array}\right\| .
$$

\section{Av mechanical power- holor representation}

An instantaneous value of the AV mechanical power can be obtained from a product

$$
p(t)=f(t) v(t) .
$$

Substituting instantaneous values of the force and velocity from Eqs (2.5) to Eq.(7.1) one obtains

$$
p(t)=F V\left\{\cos \varphi-\cos \left[2 \omega t+\left(\psi_{f}+\psi_{v}\right)\right]\right\}
$$

where

$$
\varphi=\psi_{f}-\psi_{v} .
$$


To obtain a holor representation of the mechanical power one must write Eq.(7.2) in a form

$$
p(t)=F V\left[\cos \varphi-\cos 2 \omega t \cos \left(\psi_{v^{\prime}}+\psi_{i}\right)+\sin 2 \omega t \sin \left(\psi_{u}+\psi_{i}\right)\right] .
$$

If one designed an independent term by $P^{p}$ and dependent terms from a doublet frequency by $P^{q}$ and $P^{r}$, then one obtains

$$
\begin{aligned}
& P^{p}=F V \cos \left(\psi_{f}-\psi_{v}\right)=F V \cos \varphi=P, \\
& P^{q}=F V \cos \left(\psi_{f}+\psi_{v}\right), \\
& P^{r}=F V \sin \left(\psi_{f}+\psi_{v}\right) .
\end{aligned}
$$

In this way Eq.(7.3) can be rewritten in a form

$$
p(t)=P^{p}-P^{q} \cos 2 \omega t+P^{r} \sin 2 \omega t .
$$

Quantities $P^{p}, P^{q}$ and $P^{r}$ are independent elements (merates) or co-ordinates of the three-merates monovalent holor of an AV mechanical power

$$
\boldsymbol{P}^{\kappa}=\left(P^{p}, P^{q}, P^{r}\right)
$$

this allows eliminating time from Eq.(7.5).

It results from the first expression of Eq.(7.6) that an independent element (merate) of the power holor is an AV active mechanical power, which can be measured by means of an electrodynamical wattmeter. Residual independent elements (merates) can also measured or computed with methods of electrical metrology.

In particular circumstances

a) For a force-holor phase angle $\psi_{f}=0$

$$
\begin{aligned}
& P^{q}=F V \cos \varphi=P^{p}=P ; \\
& P^{r}=-F V \sin \varphi=-Q .
\end{aligned}
$$

b) For a velocity-holor phase angle $\psi_{v}=0$

$$
\begin{aligned}
& P^{q}=F V \cos \varphi=P^{p}=P ; \\
& P^{r}=F V \sin \varphi=Q .
\end{aligned}
$$


Thus, for the force-holor phase angle $\psi_{f}=0$ or - the velocity-holor phase angle $\psi_{i}=0$, an independent element (merate) of the mechanical power holor, $P^{q}$ is the active AV mechanical power $P$, however, an independent element (merate) of the power holor $P^{r}$ is the reactive AV mechanical power $Q$. To obtain an equation, taking into account a power holor from the force holor and velocity holor one must use an adequate law of these holors multiplication.

However, in holor algebra, the conventional tensor product in the form $\boldsymbol{F}_{i} \boldsymbol{V}^{j}$, due to Moon and Spencer [5-7], cannot be applied; in the case of the mechanical power holor one uses the particularly useful univalent tensor product

$$
\boldsymbol{P}^{\kappa}=\boldsymbol{F}_{i} \otimes \boldsymbol{V}^{j}=\gamma_{. . j}^{\kappa i} \boldsymbol{F}_{i} \boldsymbol{V}^{j}
$$

where, $i, j=c, s ; \kappa=p, q, r$. A symbol $\otimes$ The univalent tensor product is marked with a symbol $\otimes$ - giving as a result a univalent holor.

Hence, applying a holor representation of the mechanical power in the AV mechanical homogeneous continuous dynamical system analysis one obtains a full analogy with the direct velocity (DV) mechanical velocity analysis. Independent elements (merates) of the mechanical power holor can be obtained from Eq.(5.4), namely

$$
P^{p}=\gamma_{. . j}^{p i} \boldsymbol{F}_{i} \boldsymbol{V}^{j}, \quad P^{q}=\gamma_{. . j}^{q i} \boldsymbol{F}_{i} \boldsymbol{V}^{j}, \quad P^{r}=\gamma_{. . j}^{r i} \boldsymbol{F}_{i} \boldsymbol{V}^{j}
$$

where

$$
\gamma_{j}^{p i}=\left\|\begin{array}{cc}
\gamma_{. . c}^{p c} & \gamma_{. . s}^{p c} \\
\gamma_{. . c}^{p s} & \gamma_{. . s}^{p s}
\end{array}\right\|=\left\|\begin{array}{cc}
1 & 0 \\
0 & 1
\end{array}\right\|, \quad \gamma_{j}^{q i}=\left\|\begin{array}{cc}
\gamma_{. . c}^{q c} & \gamma_{. .}^{q c} \\
\gamma_{. . c}^{q s} & \gamma_{. s}^{q c}
\end{array}\right\|\|\| \begin{array}{cc}
1 & 0 \\
0 & -1
\end{array}\left\|, \quad \gamma_{j}^{r i}=\right\| \begin{array}{cc}
\gamma_{. . .}^{r c} & \gamma_{. s}^{r c} \\
\gamma_{. . c}^{r s} & \gamma_{. . s}^{r c}
\end{array}\|=\| \begin{array}{cc}
1 & 0 \\
0 & 1
\end{array} \| .
$$

Applying a convention of holors summation one obtains

$$
\begin{aligned}
& P^{p}=\gamma_{. . c}^{p c} F_{c} V^{c}+\gamma_{. . s}^{p c} F_{c} V^{s}+\gamma_{. . c}^{p s} F_{s} V^{c}+\gamma_{. . s}^{p s} F_{s} V^{s}, \\
& P^{q}=\gamma_{. . c}^{q c} F_{c} V^{c}+\gamma_{. . s}^{q c} F_{c} V^{s}+\gamma_{. . c}^{q s} F_{s} V^{c}+\gamma_{. . s}^{q s} F_{s} V^{s}, \\
& P^{r}=\gamma_{. . c}^{r c} F_{c} V^{c}+\gamma_{. . s}^{r c} F_{c} V^{s}+\gamma_{. . c}^{r s} F_{s} V^{c}+\gamma_{. . s}^{r s} F_{s} V^{s} .
\end{aligned}
$$

Substituting to Eqs (7.9) independent elements (merates) of the force holor from Eq.(3.3) as well as independent elements (merates) of the velocity holor from Eqs (3.6) one obtains

$$
\begin{aligned}
& P^{p}=\gamma_{. . c}^{p c} \cos \psi_{f} \cos \psi_{v}+\gamma_{. . s}^{p c} \sin \psi_{f} \sin \psi_{v}+\gamma_{. . c}^{p s} \sin \psi_{f} \cos \psi_{v}+\gamma_{. . s}^{p s} \sin \psi_{f} \sin \psi_{v}, \\
& P^{q}=\gamma_{. . c}^{q c} \cos \psi_{f} \cos \psi_{v}+\gamma_{. . s}^{q c} \sin \psi_{f} \sin \psi_{v}+\gamma_{. . c}^{q s} \sin \psi_{f} \cos \psi_{v}+\gamma_{. . s}^{q s} \sin \psi_{f} \sin \psi_{v}, \\
& P^{r}=\gamma_{. . c}^{r c} \cos \psi_{f} \cos \psi_{v}+\gamma_{. . s}^{r c} \sin \psi_{f} \sin \psi_{v}+\gamma_{. . c}^{r s} \sin \psi_{f} \cos \psi_{v}+\gamma_{. . s}^{r s} v .
\end{aligned}
$$


From a comparison of Eqs (7.10) with Eqs (7.4) one obtains

$$
\begin{aligned}
& \gamma_{. . c}^{p c}=\gamma_{. . s}^{p s}=1, \quad \gamma_{. . s}^{p c}=\gamma_{. . c}^{p s}=0, \\
& \gamma_{. . c}^{q c}=1, \quad \gamma_{. . s}^{q s}=-1, \quad \gamma_{. . s}^{q c}=\gamma_{c}^{q s}=0, \\
& \gamma_{. . c}^{r c}=\gamma_{. . s}^{r s}=0, \quad \gamma_{. . s}^{r c}=\gamma_{. . c}^{r s}=1 .
\end{aligned}
$$

The aforementioned independent elements (merates) of a trivalent holor $\gamma$ can also be rewritten in a form of the following matrices

$$
\gamma_{. . j}^{p i}=\left\|\begin{array}{cc}
1 & 0 \\
0 & 1
\end{array}\right\|, \quad \gamma_{. . j}^{q i}=\left\|\begin{array}{cc}
1 & 0 \\
0 & -1
\end{array}\right\|, \quad \gamma_{. . j}^{r i}=\left\|\begin{array}{ll}
0 & 1 \\
1 & 0
\end{array}\right\| .
$$

Taking into account Eqs (7.10), one can express Eqs (7.9) as follows

$$
P^{p}=F_{c} V^{c}+F_{s} V^{s}, \quad P^{q}=F_{c} V^{c}-F_{s} V^{s}, \quad P^{r}=F_{c} V^{s}+F_{s} V^{c} .
$$

Routinely, for computing an AV power, an analogous expression is needed as well for computing a DV power in a form

$$
P=B\left(V^{\wedge} 2\right)
$$

From Eq.(5.4) a power holor

$$
\boldsymbol{P}^{\kappa}=\boldsymbol{F}_{i} \oplus \boldsymbol{V}^{j}=\boldsymbol{\gamma}_{. . j}^{\kappa i} \boldsymbol{F}_{i} \boldsymbol{V}^{j},
$$

however, from Eq.(3.5) a force holor

$$
\boldsymbol{F}_{i}=\boldsymbol{Z}_{i j} \boldsymbol{V}^{j}
$$

Hence, the power holor

$$
\boldsymbol{P}^{\kappa}=\boldsymbol{F}_{i} \otimes \boldsymbol{V}^{j}=\gamma_{. . j}^{\kappa i} \boldsymbol{F}_{i} \boldsymbol{V}^{j}=\gamma_{. . j}^{\kappa i} \boldsymbol{Z}_{i j} \boldsymbol{V}^{k} \boldsymbol{V}^{j}
$$

and its independent elements (merates)

$$
P^{p}=\gamma_{. . j}^{p i} \boldsymbol{Z}_{i j} \boldsymbol{V}^{k} \boldsymbol{V}^{j}, \quad P^{q}=\gamma_{. . j}^{q i} \boldsymbol{Z}_{i j} \boldsymbol{V}^{k} \boldsymbol{V}^{j}, \quad P^{r}=\gamma_{. . j}^{r i} \boldsymbol{Z}_{i j} \boldsymbol{V}^{k} \boldsymbol{V}^{j}
$$

Applying a convention of the holor summation one obtains 


$$
\begin{aligned}
& P^{p}=\gamma_{. . n}^{p m} Z_{c c}\left(V^{c \wedge} 2\right)+\gamma_{. . n}^{p m} Z_{c c} V^{c} V^{s}+\gamma_{. . n}^{p m} Z_{c s} V^{s} V^{c}+\gamma_{. . n}^{p m} Z_{c s}\left(V^{s \wedge} 2\right)+ \\
& \gamma_{. . n}^{p m} Z_{s c}\left(V^{c \wedge} 2\right)+\gamma_{. . n}^{p m} Z_{s c} V^{c} V^{s}+\gamma_{. . n}^{p m} Z_{s s} V^{s} V^{c}+\gamma_{. . n}^{p m} Z_{s s}\left(V^{s \wedge} 2\right), \\
& P^{q}=\gamma_{. . n}^{q m} Z_{c c}\left(V^{c \wedge} 2\right)+\gamma_{. . n}^{q m} Z_{c c} V^{c} V^{s}+\gamma_{. . n}^{q m} Z_{c s} V^{s} V^{c}+\gamma_{. . n}^{q m} Z_{c s}\left(V^{s \wedge} 2\right)+ \\
& \gamma_{. . n}^{q m} Z_{s c}\left(V^{c \wedge} 2\right)+\gamma_{. . n}^{q m} Z_{s c} V^{c} V^{s}+\gamma_{. . n}^{q m} Z_{s s} V^{s} V^{c}+\gamma_{. . n}^{q m} Z_{s s}\left(V^{s \wedge} 2\right), \\
& P^{r}=\gamma_{. . n}^{r m} Z_{c c}\left(V^{c \wedge} 2\right)+\gamma_{. . n}^{r m} Z_{c c} V^{c} V^{s}+\gamma_{. . n}^{r m} Z_{c s} V^{s} V^{c}+\gamma_{. . n}^{r m} Z_{c s}\left(V^{s \wedge} 2\right)+ \\
& \gamma_{. . n}^{r m} Z_{s c}\left(V^{c \wedge} 2\right)+\gamma_{. . n}^{r m} Z_{s c} V^{c} V^{s}+\gamma_{. . n}^{r m} Z_{s s} V^{s} V^{c}+\gamma_{. . n}^{r m} Z_{s s}\left(V^{s \wedge} 2\right) .
\end{aligned}
$$

Taking into consideration Eqs (5.11) and (7.11) one obtains

$$
\begin{aligned}
& P^{p}=B\left(V^{c \wedge} 2\right)+\left(V^{s \wedge} 2\right), \\
& P^{q}=B\left(V^{c \wedge} 2\right)-\left(V^{s \wedge} 2\right)-2 X V^{c} V^{s}, \\
& P^{r}=X\left(V^{c \wedge} 2\right)-\left(V^{s \wedge} 2\right)+2 R V^{c} V^{s} .
\end{aligned}
$$

Substituting Eqs (3.1) to the first expression from Eqs (3.9) one obtains

$$
P^{p}=B\left(V^{\wedge} 2\right)=P .
$$

In this way, is obtained a well-known expression for an AV mechanical continuous dynamical system analysis from which once more can be seen that an independent element (merate) of the holor power $P^{p}$ is a DV real or active power.

Adding geometrically, independent elements (merates) $P^{q}$ and $P^{r}$ of the power holor, one derives a well known expression for an AV effective value of the apparent power $S$ in a form

$$
\begin{aligned}
& \operatorname{sqrt}\left[\left(P^{q \wedge} 2\right)+\left(P^{r \wedge} 2\right)\right]=\operatorname{sqrt}\left[\left(B^{\wedge} 2\right)+\left(X^{\wedge} 2\right)\right]\left[\left(F^{c \wedge} 2\right)+\left(F^{s \wedge} 2\right)\right]= \\
& =Z\left(F^{\wedge 2}\right)=F V=S .
\end{aligned}
$$

Thus, due to the application of holor algebra for a sine-wave-input physically continuous dynamical system analysis, it is possible to have an operation with holor quantities for physically continuous dynamical systems that are analogous to each other as with real quantities for step-input physically continuous dynamical systems. 


\section{A geometrical interpretation of the force, velocity and power holors}

The aforementioned, introduced force, velocity and power holors can be geometrically represented in two- or three-dimensional (2-D or 3-D) spaces. If one assumes that independent elements (merates) of the velocity holor $V^{\mathfrak{c}}$ and $V^{\S}$ are rectangular coordinates in the 2-D space (Fig.10), then the equation of Eq. (5.5) can be regarded as an equation of the straight line $F_{\mathrm{c}}=$ const, where slope is described by $\boldsymbol{Z}_{i j}$. The second straight line $F_{\mathrm{s}}=$ const results from the other equation of Eq.(5.6).

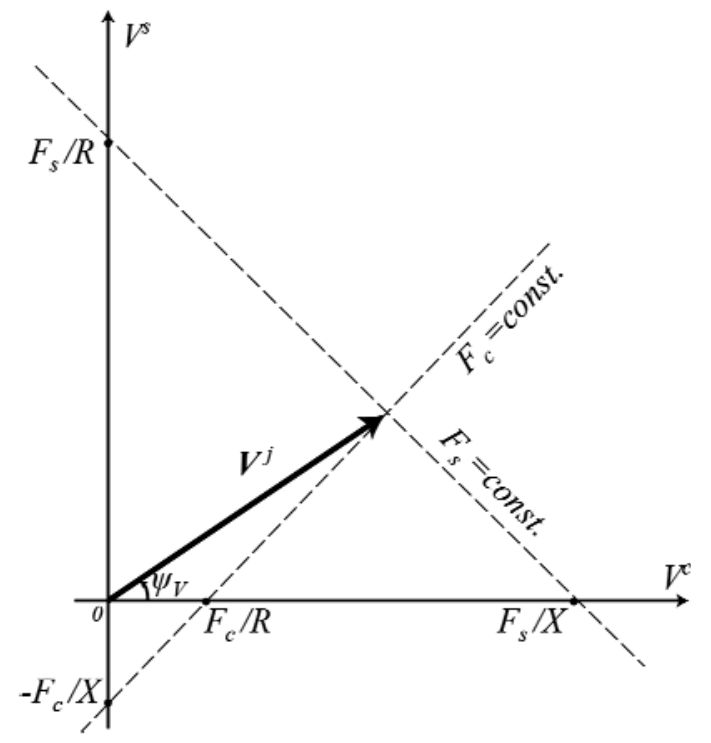

Fig.10. A geometrical representation of the velocity holor [2, 3].

An impedance holor $\boldsymbol{Z}_{i j}$ can be represented by means of a pair of the two orthogonal straight lines (see Fig.10). The velocity holor recline from a beginning of the coordinate system to cutting of each other these straight lines. In particular circumstances, for instance, for an ideal active damper, these orthogonal straight lines are running across vertically and horizontally.

The force holor may also be geometrically represented in a similar way as the velocity holor, as shown in Fig.11.

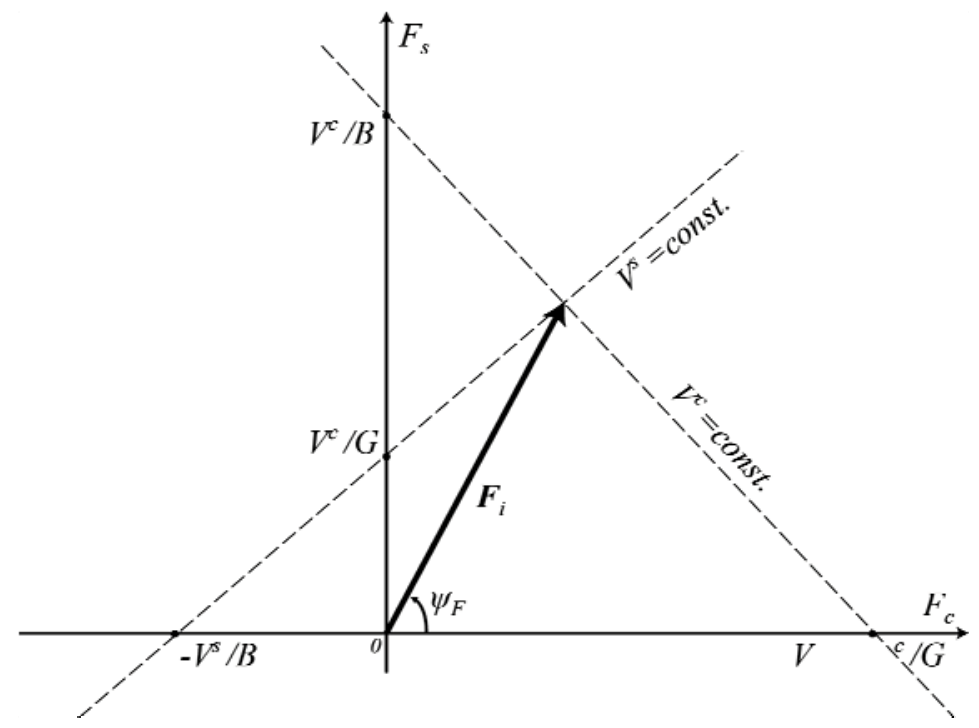

Fig.11. A geometrical representation of the force holor [2, 3]. 
A geometrical representation of the power holor needs a 3-D space, as coordinates are independent elements (merates) of the power holor $P^{p}, P^{q}$, and $P^{r}$. The coordinates $P^{q}$, and $P^{r}$ are orthogonal of each other, as regards to Eq.(7.18). The third axis $P^{p}$ established an angle $\varphi=\psi_{f}-\psi_{v}$ with a vector of the apparent power, which a module $S=F V$.

The power holor $\boldsymbol{P}^{\kappa}$ is hence a vector sum of its independent elements (merates) $P^{p}, P^{q}$, and $P^{r}$ (Fig.12).

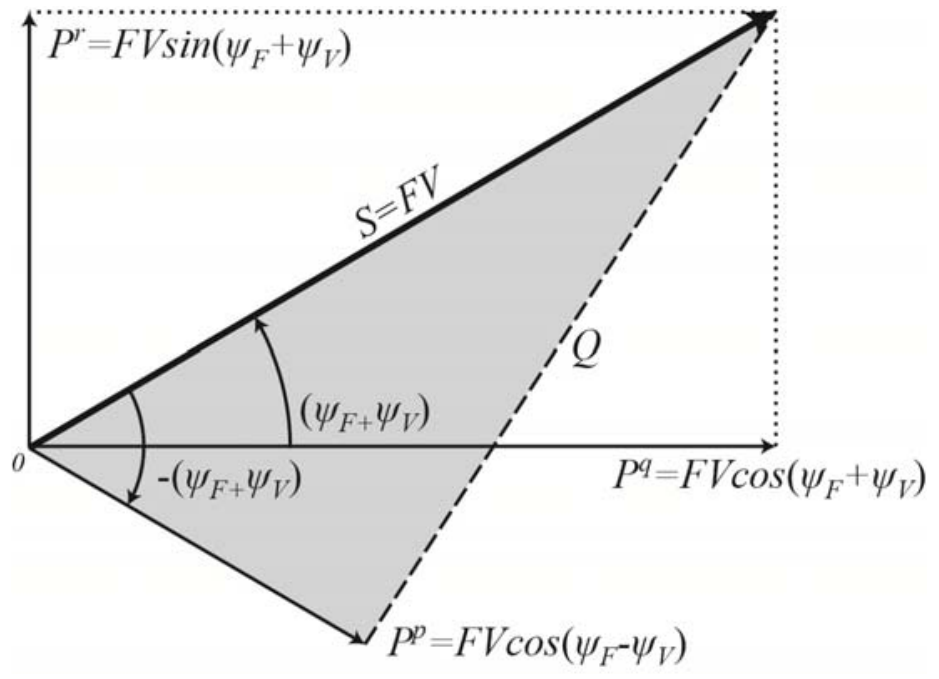

Fig.12. A geometrical representation of the power holor [2, 3].

A partial representation of the holor power needs a 2-D space. If a phase angle of the doublefrequency term is small, then a module of the double frequency power can be obtained from a part of the holor diagram shown in Fig.12, depicting a well-known holor diagram for the 'power holor' established by independent elements (merates) of the real or active power $P^{p}=P$ and the reactive power $Q$.

A partial holor diagram of the holor power, however, does not represent the holor power exactly, but a precise representation needs three independent elements (merates) and 3-D space.

Thus a physical effective value of the apparent power $P^{\mathrm{K}}$ between two terminals would be

$$
P^{\kappa}=\operatorname{sqrt}\left[\left(P^{p \wedge} 2\right)+\left(P^{q \wedge} 2\right)+\left(P^{r \wedge} 2\right)\right]=S .
$$

\section{Conclusion}

A new mathematical representation of the sinusoidal alternating velocity, force and power by means of some complex quantities, termed 'holors' is proposed. The word holor is a term to describe a mathematical entity that is made up of one or more independent quantities, and includes complex numbers, scalars, vectors, matrices, tensors and other hypernumbers [8,9]. Holors, thus defined, have been known for centuries but each has been developed more or less independently, accompanied by separate nomenclature and theory [5 - 7].

A holor algebra, similarly as a complex number algebra allows elimination of time from equations describing a physical action of the sinusoidal physical variables as well as a geometrical interpretation of the fundamental quantities of the mechanical homogeneous continuous dynamical systems as real quantities in the 2-D and 3D spaces. 
Holor algebra allows you cannot isolate of fundamental mechanical quantities into the individual terms, such as the force holor, velocity holor, impedance holor, admittance holor, and power holor.

It seems that the basic value of the holor algebra lies not only in computing processes but also in the theoretical domain, allowing a logical and approved by the traditional mathematics representation of the sinusoidal fundamental mechanical quantities.

\section{Nomenclature}

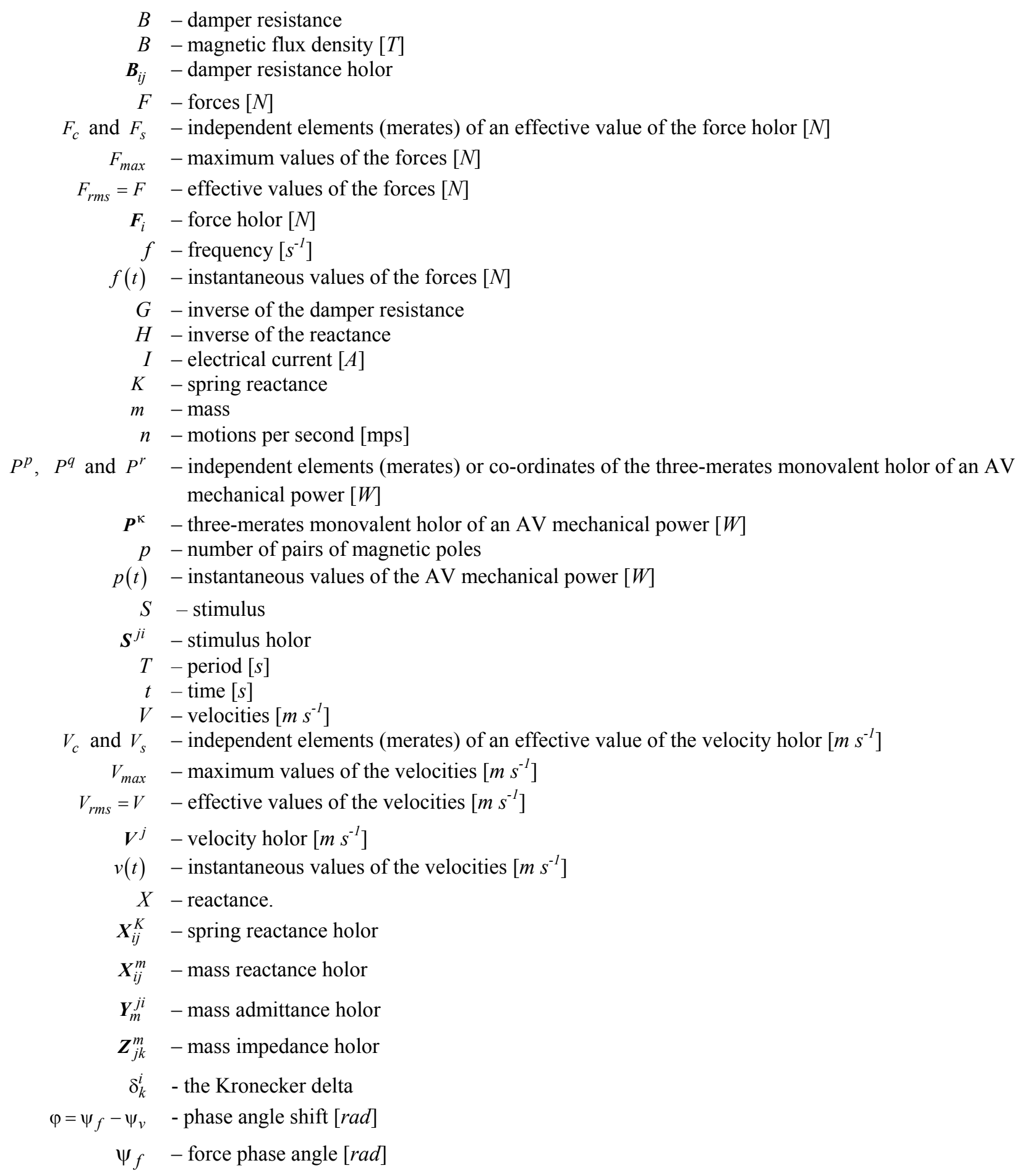




$$
\begin{aligned}
\psi_{v} & - \text { velocity phase angle }[\mathrm{rad}] \\
\omega & - \text { angular frequency }\left[\mathrm{rad} \mathrm{s} \mathrm{s}^{-1}\right] \\
\mathbb{H}^{-1}\left\{F \angle \psi_{v}\right\} \text { and } & \text { - read as the 'inverse transform' of } F \angle \psi_{f} \text { and } V \angle \psi_{v} \\
\mathbb{H}^{-1}\left\{V \angle \psi_{i}\right\} &
\end{aligned}
$$

By a symbol $\otimes$ is designed the univalent tensor product, giving as a result a univalent holor.

\section{References}

[1] H2WTech (2015): Voice Coil Actuators vs. Solenoids: What is Difference? - October 22, 2015 https:// www.h2wtech.com/article/voice-coil-actuators-vs-solenoids

[2] Fijałkowski B. (1967): An application of the holor calculus in theory of alternating currents. - Przegląd Elektrotechniczny, Zeszyt 2, pp.47-52 (In Polish).

[3] Fijalkowski B.T. (2016): Mechatronics: Towards a Unified Theory exploiting Dynamical Systems Approach and Theory of Holors. - Institute of Physics, IOP Publishing. Bristol, UK.

[4] Moon P. and Spencer D.E. (1963): A New Mathematical Representation of Alternating Currents. - Tensor, No. 14, p.110.

[5] Moon P. and Spencer D.E. (1966): Vectors. - D. van Nostrand Co., Princeton, N.Y.

[6] Moon P.H. and Spencer D.E (2009): Theory of Holors. - Cambridge: Cambridge University Press.

[7] Savant C.J. Jr. (1959): Basic Feedback Control System Design. - New York - Toronto - London: McGraw-Hill Book Company Inc.

[8] Kron G. (1939): Tensor Analysis of Networks. - Chapman and Hall Ltd.

[9] Gołąb St. (1965): Rachunek tensorowy (Tensor Calculus). - Warsaw: PWN (In Polish).

Received: August 20, 2016

Revised: September 14, 2016 